\title{
PLANETHESIZER: APPROACHING EXOPLANET SONIFICATION
}

\author{
Adrián García Riber \\ AGR Image and Sound Art, \\ Music Research and Sound Engineering, \\ Francisco Martí Mora 1-B, 22-3, IB 07011, Spain \\ adrian@imageandsoundart.com
}

\begin{abstract}
The creation of simulations, sounds and images based on information related to an object of investigation is currently a real tool used in multiple areas to bring the non-specialized public closer to scientific achievements and discoveries. Under this context of multimodal representations and simulations developed for educational and informational purposes, this work intends to build a bridge between virtual musical instruments' development and physical models, using the gravitation laws of the seven planets orbiting around the Trappist-1 star. The following is a case study of an interdisciplinary conversion algorithm design that relates musical software synthesis to exoplanets' astronomical data measured from the observed flux variations in the light curves of their star- and that tries to suggest a systematic and reproducible method, useful for any other planetary system or model-based virtual instrument design. As a result, the Virtual Interactive Synthesizer prototype Planethesizer is presented, whose default configurations display a multimodal Trappist-1, Kepler-444 and K2-72 planetary systems simulation.
\end{abstract}

\section{INTRODUCTION}

The exponential increase in virtual instruments' development and their popularity and establishment as part of the current workflow on audio productions, both in home studios and professional environments, creates a new opportunity in bringing science to the general public and designing audio tools based on physical or mathematical models of undoubted creative and artistic utility. There are numerous examples of composers who translate models based on nature or science into musical parameters to give their works a formal and structural solidity transcending their own compositional intuition. Their kind of approach, in addition to the publication of the discovery of the seven Earth-like planets orbiting around the Trappist-1 star in February 2017, inspired the idea of exploring the creation of a model-based virtual instrument. Developing an interactive astronomical data sonification tool useful in both creative and educational or informational contexts, this work also tries to suggest a possible line of interdisciplinary audio tools development.

\section{ASTRONOMICAL DATA SONIFICATION REFERENCE WORKS}

In an attempt to make a state-of-the-art review in terms of astronomical data sonification and to analyze the technology, processes, tools and techniques that are currently involved in

\section{(c) (i) (8)}

Attribution - Non Commercial 4.0 International License.

The full terms of the License are available at

http://creativecommons.org/licenses/by-nc/4.0/ this field, it seems clear that Pythagoras ( $6^{\text {th }}$ century B.C.) and his Music of the Spheres as well as Kepler's Harmonices Mundi (1619), represent the crucial starting point of the relation between occidental music and astrophysics [1]. Highly influenced by these fundamental works, the animated sonifications of the solar system from Twyman (2010) [2], and Mark Ballora's sonifications for the film Rhythms of the Universe (2013) [3] (a co-production by the ethnomusicologist and percussionist from the mythical band Grateful Dead and cosmologist George Smoot III from Lawrence Berkeley Labs), can be found. Quinton, M. et al. [4] went a step further with their perceptual research on the solar system's sonifications created for the planetarium Esplora (2016, island of Malta), proposing a planet properties test through sound recognition, realized on both specialized and non-specialized audiences. In this sense of probing the effectiveness of the astronomical data sonification processes, the work of Diaz Merced, W. L. (2013) [5], the Supernova Sonification [6] and the $x$ Sonify prototype project [7], built in collaboration with NASA, also stand out. Trying to equilibrate creative freedom and scientific accuracy to the original data sheet, it's worth mentioning the solar wind data sonifications of Alexander, R. et al. (2010) [8].

In a more creative context, Winton et al. [9] developed the sonifications of stellar data from the Kepler Telescope (2012), using Sonification Sandbox software [10] and Matlab's interpolation functions to explore the aesthetical possibilities of direct unprocessed sonifications. Also interesting are the orchestral character coding sonifications of Quinn, M. for the exoplanets and stars information database of the European Southern Observatory [11], and the mapping sonification work of Jamie Ferguson in collaboration with ESA From Hipparchus to Hipparcos (2014) [12], that translate several historic star catalogues to sound in a similar way to John Cage's Etudes Australes. Finally, it's impossible not to mention Tim Pyle's Trappist Transits musical representation (2017) [13], illustrating data from NASA's Spitzer Telescope for informational purposes.

\section{TRAPPIST-1 SYSTEM}

The Trappist-1 system is located 39 light years (12 parsecs) away from the solar system within the constellation of Aquarius. It's formed by a red dwarf star, Trappist-1A, and seven planets named from Trappist- $1 b$ to Trappist- $1 h$, as function of their radial distance to the star. In 2015 the Transiting Planets and Planetesimals Small Telescope (TRAPPIST) monitored the brightness of the star for 245 hours over 62 nights, from September 17th to December 28th, showing clear transit-like signatures later confirmed as planets $b, c$ and $d$ [14]. The system was completely identified using the Trappist telescope, the Spitzer Space Telescope, the Very Large Telescope, the UKIRT, the Liverpool Telescope and the 
William Herschel Telescope [15]. The complete system discovery was finally published in February 2017 [16].

\section{PHOTOMETRIC TRANSIT DETECTION}

The photometric transit detection technique is based on the observation of the light flux variations of celestial objects. It basically consists of pointing a telescope at the star and obtaining its light curve, a graphic representation of brightness flux variations along time. When a planet passes in front of a star, it produces a partial eclipse and the consequent loss of flux perceived can be measured in terms of time and depth. As illustrated in Figure 1, the duration of total eclipse has a direct correspondence with the decrease of energy observed in the light curve and, according to (1), the transit depth is proportional to the surface ratios of the planet and the star, $R p / R *$

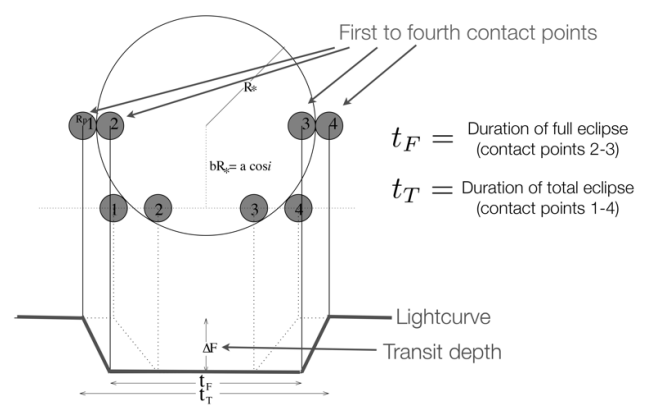

Figure 1: Measure of flux variations over a generic light curve. Seager \& Mallén-Ornelas, 2003. [17]

$$
\frac{R p}{R^{*}}=\sqrt{\Delta F}
$$

Equation (2) gives the orbital inclination of the planet, where $a$ is the orbital radius (assuming circular orbit, Kepler's $3^{\text {rd }}$ law), and $b$ is the impact parameter which represents the increase of total eclipse duration for orbits close to the star's equator. It can also be calculated from the light curve using (3).

$$
\begin{gathered}
i=\cos ^{-1} b \frac{R *}{a}, \\
b=\sqrt{\frac{(1-\sqrt{\Delta F})^{2}-\left(\frac{t F}{t T}\right)^{2}(1+\sqrt{\Delta F})^{2}}{1-\sqrt{\frac{t F}{t T}}}},
\end{gathered}
$$

The orbital period of each planet can be calculated measuring the time interval between transits. At least three transits are needed to obtain conclusive results [18].

\section{PLANETHESIZER VST PLUGIN DESIGN}

The Planethesizer prototype was designed using Iain McCurdy's works [19], LorentzSynth (2015) and Planet (2012), as an inspiration. It can be defined as a vector synthesis virtual instrument implemented from Csound's planet opcode. This opcode (or computing operation code), generates the coordinates $(x, y, z)$ of a planet orbiting a binary star system simulation whose outputs -calculated for each of the seven planets- are used to control seven software synthesizers. The instrument also provides an additional eighth synthesizer, controlled through a real-time MIDI keyboard, to improve musical interaction. Csound [20] was used in combination with Cabbage front end [21] to implement a VST (Virtual Studio Technology) plugin and maximize compatibility with any DAW (Digital Audio Workstation), both on Mac and PC computers. Blender software [22] was used for the graphic design of the star.

The prototype can be downloaded for testing from: https://archive.org/details/@agriber

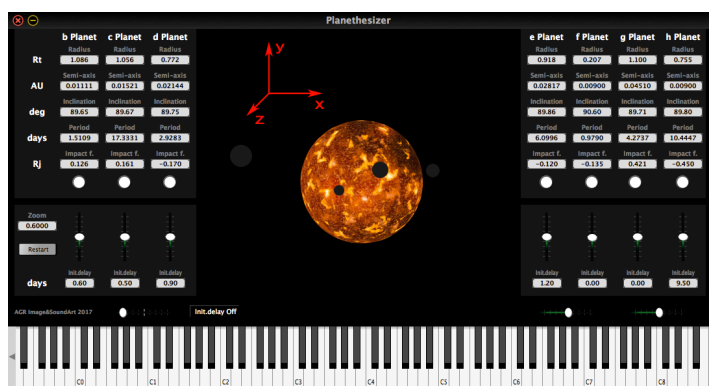

Figure 2: Planethesizer interface illustrating the axes used in the simulation's graphic display.

Figure 2 shows the prototype interface that allows endusers to control sound properties as a function of the radius, semi-axis, inclination, period and impact factor for the orbit of each planet or synthesizer in astronomical units. To reproduce the eclipse sequence cadence, each planet can be initially delayed introducing time differences expressed in days. Level control is allowed for master output, the keyboard's synthesizer as well as for every planet's module. The Restart button initializes the eclipse sequence and the Init. Delay Off button deactivates initial delay, allowing the synchronization of the planets for creative purposes. The prototype also includes a global tempo, global zoom control and one on/off button for each planet. Instrument default configuration represents a multimodal simulation of the Trappist- 1 system. Saving and recalling presets is also allowed.

According to the research agenda proposed by Kramer et al. [23], the plugin's requirements relay on three main axes: adaptation to the language and needs of the research field, enduser control or interaction and prototype integrability. As described below, several targets were defined for the prototype:

- Provide a synchronized multimodal simulation for planetary systems

- Provide an effective and interactive control for every sound parameter expressed in astronomical units

- Provide end-users a flexible control of the simulation including global tempo and single planet, keyboard and master level controls

- Develop a micro-tonal and multi-timbral musical instrument

- Develop an extremely intuitive user interface

- Develop a Trappist-1 system sonification extendable to any other planetary system

- Allow real-time operation for all parameters

- Allow full integration in Digital Audio Workstations

The plugin design process was divided, as follows, into eight blocks attending to Iannis Xenakis's 'fundamental phases of a musical work' [24]: 
1. Prototype concept definition

2. Sound elements definition

3. Physical-Musical-Mathematical algorithm design

4. Global control functions definition

5. Simplified prototype implementation, $b$ planet

6. Full prototype implementation

7. Default configuration and presets implementation

8. Exporting to final format and testing under different DAW's

\section{IMPLEMENTATION ALGORITHM}

In order to maximize the intelligibility and simplicity of calculation, in Planethesizer every sound appears linked to each planet's graphic simulation, forming a multimodal representation with a high degree of intelligibility that minimizes its learning curve. Figure 3 describes the interactive algorithm, parameters and variables used to control the planets' simulation. As shown in the block diagram, the planet opcode generates three-dimensional vectors whose coordinates ( $a x, a y, a z)$ can be manipulated in real-time by end-users. Delayed variables ( $a x d, a y d, a z d)$, are scaled and converted to Csound's control variables $(k x, k y, k z)$ to achieve the final visual representation and to allow synchronized sonification.

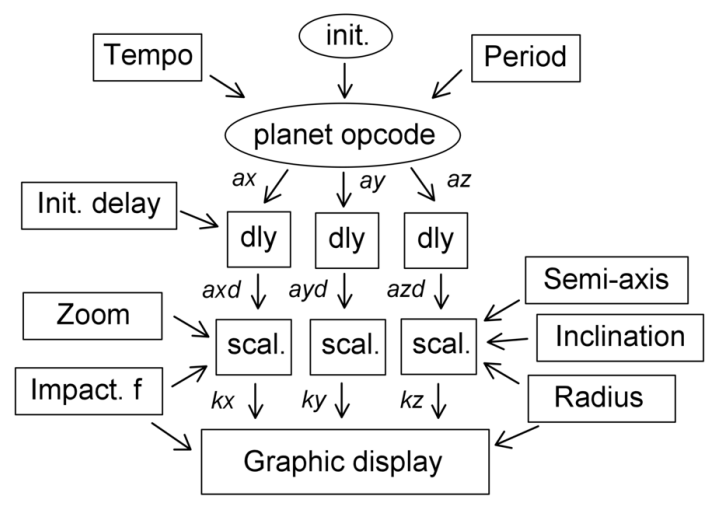

Figure 3: Block diagram of the synthesizer's control algorithm.

Global Tempo control changes the speed of the complete eclipses sequence. Initial delay creates the desired eclipses time sequence in concordance to observed transits, as described below in Table 1. Zoom modifies all planet size and amplitude to increase realism and interactivity. Impact.f is commonly expressed in units related to Jupiter's radius and in the prototype, positive factors represent orbits above the star's equator. Negative factors were used for $d, e, f$ and $h$ planets as a uniformity convention. Orbital Period is measured in days. Semi-axis is measured in astronomical units (distance Earth$\mathrm{Sun}=1 \mathrm{AU}=14,959,7871 \mathrm{Km})$. Inclination has a multiplying factor that makes the simulation differ from observed data. For aesthetical reasons, 90 degrees correspond to horizontal axis. The planet's Radius control modifies both the timbre or pitch and size of the planet representation and its measure is related to Earth's radius.

As described in the Canonical Csound Reference Manual [25], planet opcode uses eleven arguments to generate the above-mentioned output coordinates (ax, ay, az). These arguments were initialized as follows:
- kmass 1: Represents the mass of the first star in the simulated binary star system.

- kmass2: Represents the mass of the second star.

- ksep: Represents distance between the two stars.

After intense testing with a one planet simplified prototype, kmass 1 and kmass 2 values were respectively fixed to $11,60.458$ and 0.001 , adapting the opcode capabilities to the prototype's simulation needs.

- $i x, i y, i z$ : Introduce the initial coordinates of the planet. Initializing $i x$ to -1 , the planet's rotation is set counter clockwise. 0.1 and 0.3 values were fixed to $i y$ and $i z$ in prototype's behavior optimization process.

- ivx, ivy, ivz: Allow to introduce the initial vector's speed and were set to 0,0 and 3.35 to assure stability.

- idelta (ih): Controls the model's velocity as function of orbital period divided by 100,000 . This factor was introduced to maintain planet's visualization.

- ifric: Introduces a gradual attenuation into planet's orbit. It was initialized to zero to achieve the perpetual intended simulation.

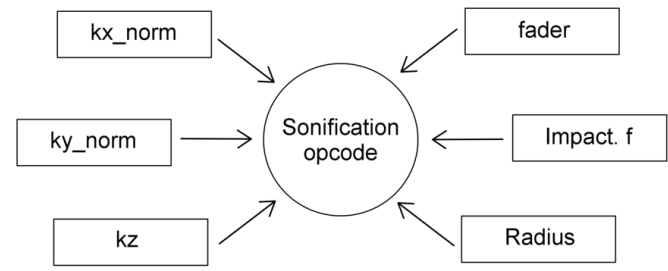

Figure 4: Block diagram showing the six parameters involved in each planet sonification. Note that $k x$ and $k y$ were normalized to assure sound stability.

As a descriptive reference point, the Trappist-1 sonification's left-placed planets represent lowest frequency sounds, analogously to the frequency axis of spectral analysis or the left hand in keyboard instruments. Sound duration was related to the visible hemicycle of the orbits and therefore to orbital period and $x$ coordinate in the visual representation ( $k x$ norm Csound's variable). Global tempo also modifies this variable. Pitch was related to $y$ coordinate ( $k y$ norm Csound's variable), in order to achieve a highly intuitive design and to ease the planets' auditory recognition. Highest sounds correspond to highest $y$ values and by modifying the orbit's inclination control, tonal sweeps are generated. The $z$ coordinate ( $\mathrm{kz}$ Csound's variable) was related to sound intensity allowing a fade in and fade out synchronized audiovisual representation. Global zoom and planets' faders also affect this variable. The following magnitudes were dismissed in the implementation of the plugin but, if needed, they could be included in future prototypes:

- The Surface temperature of each planet could be colorcoded for visual differentiation and their Irradiation could be related to the opacity of the representations.

- The Mass and Density could be mapped to the amount of an additional sound effect such as flanger or phaser.

- The Eccentricity was delegated on planet opcode, assuming circular orbits.

- The transits duration doesn't correspond to observed data since the star simulation is not affected by zoom control. 
Intensive testing with sound generation opcodes described in Csound's Manual [25] was also done. Final choice required a compromise solution between processing CPU load and sound richness. Finally, the Csound's generators shown on Table 1 were chosen to create a multi-timbral instrument and to increase clarity and precision in the visual to sound association of the multimodal display. Different timbres have been searched with the exception of planets e, f and g. These planets generate their sound using identical oscillators whose fundamental frequency can be controlled acting on the radius parameter. This makes possible the creation of micro-tonal chords expanding the synthesizer's creative possibilities.

Table 1. Csound opcodes, synthesis types and initial delay (in days), used for Trappist-1 System Sonification.

\begin{tabular}{|c|c|c|c|}
\hline Planet & Opcode & Synthesis & Delay \\
\hline b & fof & Granular & 0.6 \\
\hline c & $\begin{array}{c}\text { vco2 } \\
\text { Moogladder }\end{array}$ & $\begin{array}{c}\text { Wavetable } \\
\text { LPF }\end{array}$ & 0.5 \\
\hline d & buzz & Harmonic sinusoids & 0.9 \\
\hline e & poscil3 & Hi resolution sinusoid & 1.2 \\
\hline f & poscil3 & Hi resolution sinusoid & 0 \\
\hline g & poscil3 & Hi resolution sinusoid & 0 \\
\hline h & hsboscil & Wavetable & 9.5 \\
\hline Keys & foscil & FM & - \\
\hline
\end{tabular}

To create the desired simulated planetary sequence, a time conversion from seconds (inside the simulation) to days (in observed data) was needed. This conversion was made comparing the duration of 15 complete periods of different planets in the prototype and time intervals between observed Trappist- 1 exoplanets shown in Table 1. As a result, an initial delay, expressed in days, can be entered for each planet of the prototype in concordance with orbital period units. This delay can be globally switched off for creative purposes.

The next tables show the mapping conversion used for the opcodes listed in Table 1. Opcode's arguments are fully described in the Canonical Csound's Reference Manual [25]. For a complete analysis, Planethesizer's source code is available through Cabbage or Csound software installation.

Table 2. Conversion used in $b$ planet sonification.

\begin{tabular}{lr}
\hline fof opcode arguments & Mapping value \\
\hline xamp & $2 *$ kamp*kvol*kMast \\
xfund & cpsmidinn (knum) \\
xform & cpsoct $($ kcfoct $)$ \\
koct & $\mathrm{kb}$ \\
kband & $100 *(\mathrm{kRp})$ \\
kris/kdur/kdec & $0.002 *(\mathrm{kRp}) / 0.2 / 100$ \\
\hline
\end{tabular}

Several of Csound's control variables and functions were used during the prototype implementation process. As shown on Tables 2 to 7, to introduce amplitude, fader and master level control in the opcodes' amplitude argument (xamp and kamp) respectively kamp, $\mathrm{kvol}$ and $\mathrm{kMast}$ variables were used. Impact factor control is introduced by $k b$ variable to control octave (koct), fundamental frequency (kcps and xcps) or the number of harmonics ( $k n h$ in d planet's buzz opcode) synthesized. The planet's Radius is represented by $k R p$ and introduces sound character or pitch variations. Some factors were also applied in the algorithm to optimize the simulation. In order to maintain auditory display under a controlled audio frequency range, knum and kcfoct are respectively scaled versions of $k x \_n o r m$ and $k y \_n o r m$. The cpsmidinn and cpsoct functions convert knum and $k c f o c t$ values to cycles per second, allowing to control the fundamental frequency and octave range of the generated sound.

Table 3. Conversion used in c planet sonification.

\begin{tabular}{lr}
\hline vco2 opcode arguments & Mapping value \\
\hline kamp & 2 kamp*kvol*kMast \\
kcps & cpsmidinn(knum) \\
imode & 2 \\
inyx & 0.5 \\
\hline
\end{tabular}

To introduce the planet's impact factor $(k b)$ in the generator's fundamental frequency control of buzz opcode, kcps variable was defined as $\mathrm{kx}$ norm*90*(2+kb). Table 4 illustrates how planet's Radius ( $k R p)$, also modifies this frequency.

Table 4. Conversion used in $d$ planet sonification.

\begin{tabular}{|c|c|}
\hline buzz opcode arguments & Mapping value \\
\hline xamp & $2 *$ kamp*kvol*kMast \\
\hline xcps & $\mathrm{kcps} * 2 * \mathrm{kRp}$ \\
\hline $\mathrm{knh}$ & $20 * \mathrm{ky} \_$norm $*(2+\mathrm{kb})$ \\
\hline ifn & gisine \\
\hline
\end{tabular}

Planets $e$ and $f$ use identical mapping so their sound differences in the Trappist-1 system simulation are exclusively due to the values of the planets' astronomical data. As can be experimented in the prototype soloing these planets, this kind of approach is highly recommended if auditory display properties differentiation is a must.

Table 5. Conversion used in $e$ and $f$ planets.

\begin{tabular}{lr}
\hline poscil3 arguments & Mapping value \\
\hline kamp & kamp*kvol*kMast \\
kcps & $50 *(2+\mathrm{kb}) * \mathrm{kRp} / \mathrm{ky}$ _norm \\
ifn & \\
iphs & ftgenonce $1,0,4096,10,1$ \\
\hline
\end{tabular}

In the sonification of $g$ planet, the same poscil3 opcode was used. A multiplication factor was introduced to obtain one octave higher representation.

Table 6. Conversion used in $g$ planet sonification.

\begin{tabular}{|c|c|}
\hline poscil3 arguments & Mapping value \\
\hline kamp & kamp*kvol*kMas \\
\hline kcps & $100 *(2+\mathrm{kb}) * \mathrm{kRp} / \mathrm{ky}$ norm \\
\hline ifn & $\begin{array}{lll}2 & -1 & -1\end{array}$ \\
\hline iphs & ftgenonce $1,0,4096,10,1$ \\
\hline
\end{tabular}

The tone (ktone) and brightness (kbrite) of the last planet sonification needed to be scaled to assure sound stability.

Table 7. Conversion used in $h$ planet sonification.

\begin{tabular}{lr}
\hline hsboscil arguments & Mapping value \\
\hline kamp & kamp*kvol*kMast \\
ktone & Between -4 and $40 * \mathrm{~kb}$ \\
kbrite & Between $-2 * 1.6 / \mathrm{kRp}$ and 14 \\
ibasfreq & 100 \\
ioctfn & 0 \\
iphs & 2 \\
\hline
\end{tabular}

Table 8. Conversion used for MIDI keyboard.

\begin{tabular}{lr}
\hline foscil arguments & Mapping value \\
\hline xamp & iamp*kenv (MIDI, p5) \\
kcps & icps (MIDI, p4) \\
xcar & 1 \\
xmod & 1.414 \\
knxd & 2 \\
ifn & 1 \\
\hline
\end{tabular}




\section{SIMULATED VS MEASURED PLANETARY SEQUENCE}

Once the prototype was completely implemented, the sonification of the Trappist-1 system was recorded and analyzed to test its behavior. The spectrograms of Figure 5 obtained with Sonic Visualizer software [26], show the audio file content in frequency (vertical axis) and time (horizontal), with time in seconds and a frequency range of $80 \mathrm{~Hz}$ to $22 \mathrm{Kz}$ in the upper graph, and $10 \mathrm{~Hz}$ to $1.5 \mathrm{KHz}$ in the low frequency detail spectrogram. The transit moments of each planet around Trappist-1 star are also shown in the lower graph of Figure 5. Here, horizontal axis corresponds to time in days and every planet is represented in different colors and lines, suggesting the correspondence of each transit with the generation of a sound in a clear analogy to musical notation. This information was obtained by the NASA Spitzer Space Telescope (SST) in 2016 [27], measuring the star light intensity variations, almost continuously for 500 hours.
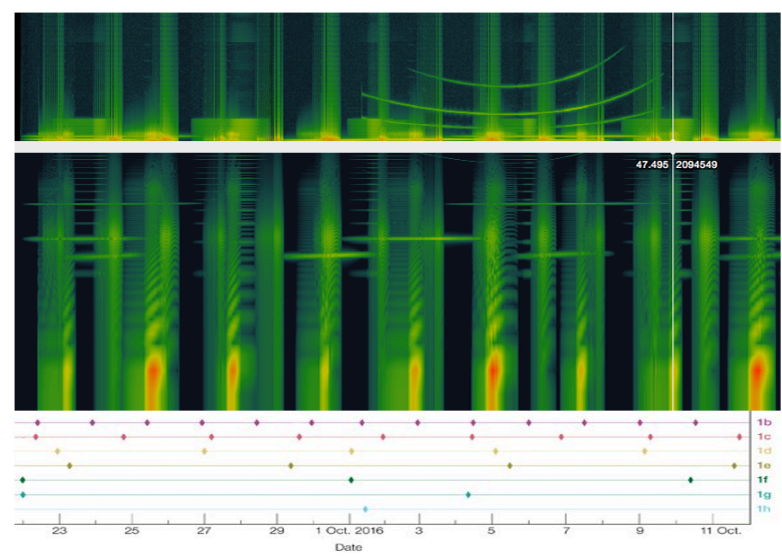

Figure 5: Comparison between the simulated eclipse sequence and the Trappist-1 system telescope observations published by Guillon, M. et al., 2017. Both spectrograms (top and middle) are related to same audio file and its frequency axis was split for a complete recognition of sound events.

The comparative image shown on Figure 5 allows us to appreciate how the simulated sequence matches the behavior of the observed exoplanets system -assuming no precision- a fact that suggests the possibility of predicting transit moments from the simulation prototype beyond the observed data, which might be useful for informative purposes.

Analyzing the spectrograms, planet $h$, with a single transit and a concave curve spectral representation can be easily distinguished. It only appears in the upper spectrogram as its fundamental frequency exceeds the limit used in the lower representation. With two transits, Planet $g$ is also easily identifiable as two narrow horizontal straight lines in the upper area of the low frequency graph. The length of these lines, equivalent to sound duration, corresponds approximately to half of its orbital period (sounds have an envelope of amplitude with a certain time of attack and relaxation that can reduce accuracy). Since the sonifications of planet $f$ and $e$, with three and four transits respectively, were performed with the same synthesizer as $g$, their representations also appear as straight lines whose vertical position depends on the fundamental frequency assigned by radius and impact factor parameters. Likewise, the five transits of planet $d$ can be recognized as columns of spaced horizontal lines corresponding to its synthesized harmonics. On the other hand, sounds generated by planets $c$ and $b$ are difficult to identify in the spectrogram due to their time coincidence and their similar spectral representation. Both sonifications generate sinusoidal pulses, represented as vertical columns that occupy the entire frequency range. The two planets are, however, easily distinguishable by listening to their sound representation.

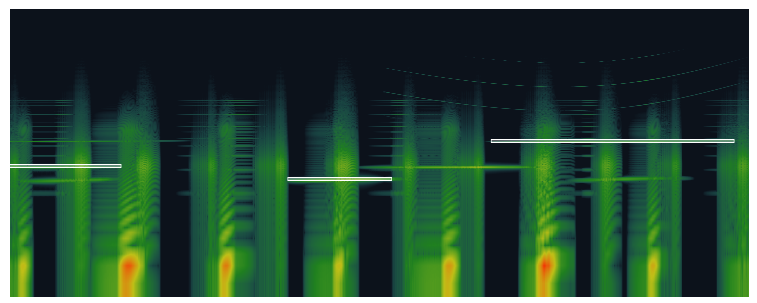

Figure 6: Trappist-1 sonification detail showing pitch cues of planets $e, f$ and $g$. Respectively 157. $968 \mathrm{~Hz}(\mathrm{D} \# 3+27 \mathrm{c})$, $236.685 \mathrm{~Hz}(\mathrm{A \# 3}+27 \mathrm{c})$ and $487.241 \mathrm{~Hz}(\mathrm{~B} 4-23 \mathrm{c})$. Sonic Visualizer software.

Finally, it's important to mention that the intense orange shapes appearing in the spectrogram correspond to sound energy accumulation and should not be interpreted as any additional event. It is also necessary to mention how the activation of the sounds doesn't match that of maximum amplitude for reasons of concordance with the visual representation, so these areas have no meaning outside the prototype.

\section{APLYING THE MODEL TO OTHER PLANETARY SYSTEMS}

In order to verify the usefulness of the prototype in the representation of planetary systems, and in addition to the default configuration of the Trappist-1 system, two presets have been incorporated representing respectively the Kepler planetary systems 444 and K2-72. Both planetary systems were observed by the Kepler space telescope using the photometric transit detection method and have been selected from the NASA's official file of confirmed exoplanets [28].

The Kepler-444 system is formed by five Eath-like planets orbiting around its star with periods comprised between 3,6 and 9,74 days. It is estimated that it was formed when the universe was $20 \%$ of its current age so is the oldest known Earth-like planetary system. All the information about this system can be found in the article by Campante et al. [29]. K2-72 is a system formed by four Earth-like planets orbiting EPIC 206209135. It is estimated that the planet $\mathrm{K} 2-72 \mathrm{~d}$ is $10 \%$ warmer than Earth while K2-72e could be $6 \%$ colder than our planet [30].

This evaluation of the prototype's possibilities revealed some considerations to take into account. In order to represent any planetary system, the scale of the variables involved in the simulation must be reconfigured attending to their astronomical dimensions. The above-mentioned planetary systems were chosen from NASA's file to fit the initial simulation model, as some other planetary systems were out of range. To solve this issue, an end-user's interactive variable range control is recommended for future versions or designs. The time-delay based eclipse sequence generator proposed in the prototype was validated in all cases. The sound representation proposed allows us to establish clear comparison between orbital periods and semi-axes of different planetary systems, but to satisfy any end-user's comparative 
requirements, an interactive synthesizer selection control should be implemented in future versions or designs.

\section{PERCEPTUAL ASPECTS}

According to Vogt's approaches [31], the task of human auditory system consists in segregating and fusing the frequency components in correspondence with real-world phenomena. This fusion can occur due to the proximity of sounds' amplitude, time, frequency, periodicity or spatial localization and generates masking effects, widely studied in Psychoacoustics reference literature listed by De Campo [32]. Other aspects, such as context in which a sound is generated or timbre similarity of sound elements, can also produce associations in the auditory system. These groups can sometimes be desirable, like in the case of generating musical chords, or generate confusion and loss of information, as in the case of a conversation masked by an ambulance's siren. While analyzing and designing sonification tools, these associations may cause loss of information. In addition, subjectivity in sound perception is presented as one of the greatest obstacles to overcome in the development of sonification tools and its importance as a cognitive process is often overlooked due to its everydayness.

Planethesizer's sonification design was, therefore, oriented to provide a clear auditory distinction of each periodic sound event represented in the simulation, with the exception of planets $b$ and $c$, whose intentional similarity was focused to test their graphical recognition through spectrogram analysis. Despite using similar oscillators -sinusoid pulses- in both cases, whose spectrograms are difficult to differentiate in time coincidence situations, auditory recognition is possible thanks to the perception of their fundamental frequency, demonstrating that auditory representations offer a useful complementary source of information to graphical display. As suggested by Walker and Brewster [33], the spatialization of sound sources also facilitates the interpretation of information. Attending to this idea, a stereophonic representation was chosen for the prototype, in which the recreated stereo image width varies as function of the planets' orbital radius.

With the intention of providing a didactic example for masking effects both in graphic and auditory displays, the three identical oscillators of planets $e, f$ and $g$ can be tuned to desired frequencies modifying the corresponding radius or impact factor of each planet. In this way, a chord of three notes can be generated, allowing a 'desirable' fusion of the sounds. Starting from identical time configurations for the three planets, a synchronized sound block is generated allowing the observation of how the graphic display loses its usefulness, despite the auditory display still providing a clear differentiation of the sounds that conform the chord. In a similar way, the 'Cocktail party effect' can be experimented representing the entire system or focusing attention on a specific planet. The prototype also allows 'Haas effect' monitoring acting over initial delay on two unison sounding planets. Chords, Arpeggio, Unison, Cocktail and Haas presets were included in the prototype for this application. As a creative musical add-on, it's also possible in the prototype to generate real time arpeggios introducing an initial delay or switching the On/Off button of the desired modules. Musical figures can also be made by controlling the planets' speed through orbital period or global tempo alterations.

\section{CONCLUSION AND PROSPECTIVE}

Throughout the development of this research work, an intense approximation to the techniques and processes related to astronomical data sonification was made. The analysis of several works carried out in this field resulted crucial to understanding the problem of sound representation and its application in scientific research. However, the comparison and evaluation of projects of this nature is complex due to the amplitude and heterogeneity of the parameters involved. Under this perspective, and despite the fact that sonification processes are in full swing, it is necessary to invest resources, time and effort in the development of new tools that encourage the expansion of this field and its countless applications. Frauenberger, C. [34] also claims the need to demystify the process of designing sound representations to establish the auditory channel as an appropriate interactive medium in the transfer of knowledge.

One of the greatest difficulties found in the development of sonification tools lies in the conjunction of multiple interdisciplinary aspects required to ensure the final usefulness of the prototype. From this point of view, the possibility of using opcodes, frameworks and accessible documentation for their use alleviated the project burden in an important way. As Stephen Barras expresses in his golden rules for the design of sound representations [35], through the experimental development of the prototype, it was verified how most direct representations require less psychological descriptions. In this sense, vector synthesis from a graphic representation's coordinates, offered a direct and complete way of representation for the basic description of the model under study. Likewise, the multimodal representation achieved complies with the 'level principle' (Cleveland WS, 1985, cited in [35], chapter 13, p.7), which expresses how the potential of a representation is a function of its ability to summarize a general behavior, while allowing examination in detail.

Although it has been necessary to delimit the ranges of values of some parameters to maintain the internal coherence of the simulation, these restrictions are easily eliminated or adaptable, if a new relation of input data is required. Several compromise solutions that distance the simulation from reality were made in order to provide an intuitive interface for nonexpert users and to add creative facilities to the prototype. It is worth mentioning here the case of the orbital inclination, oversized to magnify its response or the impact factor sign interpretation. These deviations should be considered descriptive purpose licenses that can also be easily eliminated if needed. Giving final-users the ability to choose the type of synthesis and range of values of each sound parameter, would imply an interesting improvement of the prototype providing the synthesizer with total interactivity.

Planethesizer virtual instrument development has finally served to confirm the possibilities of multimodal representations and sonification as a discipline capable of actively contributing to the development and expansion of interdisciplinary knowledge and creativity. It provides a range of functionalities that extend its range of application beyond the simulation of planetary systems and the generation of musical sequences, allowing the creation of micro-tonal chords and even its use for educational or informational purposes related to astronomy, exoplanet detection, sonification processes, musical perception or audio synthesis. 


\section{ACKNOWLEDGMENT}

Special thanks to Edith Alonso Sánchez for the support and guidance in the research work on which this document is based [36], and to Carmen Enrique and Helen Gräwert for their clinical eye.

\section{REFERENCES}

[1] García Martín, R. (2009). La teoría de la armonía de las esferas en el libro quinto de Harmonices Mundi de Johannes Kepler. (Doctoral Thesis). Universidad de Salamanca.

[2] Twyman, L. (2010). Retrieved on 05/01/2017 from: $\mathrm{http} / / /$ whitevinyldesign.com/solarbeat/2010

[3] Ballora, M. (June, 2014). Sonification strategies for the film Rhythms of the Universe. Proceedings of the $20^{\text {th }}$ International Conference on Auditory Display, New York, USA.

[4] Quinton, M., Mcgregor, I. and Benyon, D. (July, 2016). Sonifying the Solar System. Proceedings of the $22^{\text {nd }}$ International Conference on Auditory Display, Canberra, Australia.

[5] Diaz Merced, W. L. (2013). Sound for the exploration of space physics data. (Doctoral Thesis). University of Glasgow.

[6] Diaz Merced, W. L. (2014). Supernova Sonification. Retrieved on $0525 / 2017$ from:

http://www.npr.org/sections/thetwo-

way/2014/01/10/261397236/dying-stars-write-their-ownswan-songs

[7] Diaz-Merced, W. L., Candey, R.M., Brickhouse,N., Schneps, M., Mannone, J.C., Brewster, S. and Kolenberg, K. (2012). Sonification of Astronomical Data. New Horizons in Time-Domain Astronomy Proceedings IAU Symposium No. 285, 2011. R.E.M. Griffin, R.J. Hanisch \& R. Seaman, eds.

[8] Alexander, R., Zurbuchen, T. H., Gilbert, J., Lepri, S. and Raines, J. (2010). Sonification of AC Level 2 Solar Wind Data. Proceedings of the $16^{\text {th }}$ International Conference on Auditory Display, Washington D.C., USA.

[9] Winton, R., Thomas M. Gable, T. M., Jonathan Schuett, J. y Walker, B. N. (June 2012). A sonification of Kepler space telescope star data. Proceedings of the $18^{\text {th }}$ International Conference on Auditory Display, Atlanta, GA, USA

[10] Walker, B. N. (2010). Sonification Sandbox (Version 6.1) [Software]. Atlanta, GA: Georgia Institute of Technology.

Retrieved from:

http://sonify.psych.gatech.edu/research/sonification_sand box

[11] Quinn, M. Retrieved on 05/01/2017 from: http://drsrl.com

[12] Ferguson, J. (2015). From Hipparchus to Hipparcos: A Sonification of Stellar Catalogues. Retrieved from: http://jfergusoncompsci.co.uk/research
[13] Pyle, T. (2017). Trappist Transits. Retrieved on May 24 from: http://www.spitzer.caltech.edu/explore/blog/371Making-Music-from-Exoplanets

[14] Gillon, M., Jehin, E., Lederer, S. M., Delrez, L., De Wit, J., Burdanov,A., Van Grootel, V., Burgasser, A. J., Triaud, A. H. M. J., Opitom, C., Demory, B., Sahu, D. K., Gagliuffi, D. B., Magain, P. and Queloz, D. (2016). Temperate Earth-sized planets transiting a nearby ultracool dwarf star. Nature, Vol 533, pp. 221-223

[15] Trappist one, 2017, accessed June 2017, http://www.trappist.one/\#about

[16] Gillon, M., Jehin, E., Lederer, S. M., Delrez, L., De Wit, J., Burdanov,A., Van Grootel, V., Burgasser, A. J., Triaud, A. H. M. J., Opitom, C., Demory, B., Sahu, D. K., Gagliuffi, D. B., Magain, P. and Queloz, D. (February 2017). Seven temperate terrestrial planets around the nearby ultracool dwarf star TRAPPIST-1. Nature, Vol 542, pp. $456-460$

[17] Waldmann, I. 2013, Finding exoplanets, the Transit technique. Accessed June 2017, http://studylib.net/doc/8188000/111--finding-exoplanetsthe-transit-technique

[18] Planet Hunters Guide, NASA, accessed May 2017, https://www.planethunters.org/\#/education

[19] Iain McCurdy, accessed March 2017, http://iainmccurdy.org/

[20] CSound software, accessed February 2017, http://www.csounds.com

[21] Cabbage software, accessed January 2017, http://cabbageaudio.com

[22] Blender software, accessed March 2017, https://www.blender.org

[23] Kramer, G., Walker, B., Bonebright, T., Cook, P., Flowers, J. H., Miner, N. and Neuhoff, J. (2010). Sonification Report: Status of the Field and Research Agenda. Faculty Publications, Department of Psychology. Paper 444. Retrieved from: http://digitalcommons.unl.edu/psychfacpub/444

[24] Xenakis, I. (1992). Formalized Music. Thought and Mathematics in Music. Hillsdale, NY: Pendragon Press.

[25] Vercoe, B. (May, 2017). The Canonical Csound Reference Manual. Retrieved on 06/22/2017 from: http://csound.github.io/docs/manual/index.html

[26] Cannam, C., Landone, C. and Sandler, M. (2010). Sonic Visualiser: An Open Source Application for Viewing, Analysing, and Annotating Music Audio Files. Presentado en Proceedings of the ACM Multimedia International Conference, Firenze, Italia.

[27] http://www.trappist.one/\#plots

[28] NASA. (2017). Exoplanets archive. Retrieved on $07 / 20 / 2017$ from: https://exoplanetarchive.ipac.caltech.edu/cgibin/TblView/nphtblView?app=ExoTbls\&config=planets 
[29] Campante, T. L. y Barclay, T., Swift5, J. J., Huber, D., Adibekyan, V. Zh., Cochran, W., Burke, C. J.,Isaacson, H., Quintana, E. V., Davies, G. R., Silva Aguirre, V., Ragozzine, D., Riddle, R., Baranec, C., Basu, S., Chaplin, W. J., Christensen-Dalsgaar, J., Metcalfe, T. S., Bedding, T. R., Handberg, R., Stello, D., Brewer, J. M., Hekker, S., Karo, C., Kolbl, R., Law, N. M., Lundkvist, M., Miglio, A., Rowe, J. F., Santos, N. C., Van Laerhoven, C., Arentoft, T., Elsworth, Y. P., Fischer, D. A., Kawaler, S. D., Kjeldsen, H., Lund, M. N., Marcy, G. W., Sousa, S. G., Sozzetti, A., White T. R. (2015). An ancient extrasolar system with five sub-Earth-size planets. Astrophysical Journal. Retrieved on 07/16/2017 from: https://arxiv.org/abs/1501.06227

[30] Dressing, C. D., Schlieder, J.E., Crossfield, I.J.M., Newton, E. R., Ciardi, D.R., Fulton, B. J., Gonzales, E.J., Howard, A. W., Isaacson, H., Livingston, J., Petigura, E. A., Sinukoff, E., Everett, M., Horch, E. and Howell, S.B. (2017). Characterizing K2 candidate planetary sistems orbiting low-mass stars II: Planetary sistems observed during campaigns 1-7. Cornell University Library. Retrieved on 07/20/2017 from: https://arxiv.org/abs/1703.07416
[31] Vogt, K. (June, 2010). Sonification of Simulations in Computational Physics. (Thesis). Institute for Electronic Music and Acoustics, University of Music and Performing Arts, Graz, Austria.

[32] De Campo, A. (February, 2009). Science by ear. An interdiciplinary Approach to Sonifying Scientific Data. (Thesis). University for Music and Dramatic Arts, Graz.

[33] Walker, A. y Brewster, S. (2000). Spatial Audio in small screen device displays. University of Glasgow, Scotland. Retrieved on 06/24/2017 from: http://eprints.gla.ac.uk/3205/1/spatial_audio1.pdf

[34] Frauenberger, C. (July, 2009). Auditory Display Design. (Thesis). Queen Mary University of London.

[35] Boulanger, R. (Ed.) (2000). The Csound Book: Perspectives in Software Synthesis, Sound Design, Signal Processing, and Programming. Cambridge, MA, USA: MIT Press.

[36] García Riber, A. (October, 2017). Planethesizer: Aproximación a la sonificación de exoplanetas. (Master's Thesis). International University of La Rioja. 